Pattern Recognition Letters

journal homepage: www.elsevier.com

Preprint

http://dx.doi.org/10.1016/j.patrec.2017.05.006

\title{
Exploiting line metric reconstruction from non-central circular panoramas
}

\author{
Jesus Bermudez-Cameo ${ }^{\mathrm{a}, * *}$, Olivier Saurer ${ }^{\mathrm{b}}$, Gonzalo Lopez-Nicolas ${ }^{\mathrm{a}}$, Jose. J. Guerrero ${ }^{\mathrm{a}}$, Marc Pollefeys ${ }^{\mathrm{b}}$ \\ ${ }^{a}$ Instituto de investigación en Ingeniería de Aragón - Universidad de Zaragoza, C/María de Luna, 1, Zaragoza, 50018, Spain \\ ${ }^{b}$ ETH Zurich, Universitatstrasse 6, Zurich, 8092, Switzerland
}

\section{ABSTRACT}

In certain non-central imaging systems, straight lines are projected via a non-planar surface encapsulating the 4 degrees of freedom of the 3D line. Consequently the geometry of the 3D line can be recovered from a minimum of four image points. However, with classical non-central catadioptric systems there is not enough effective baseline for a practical implementation of the method. In this paper we propose a multi-camera system configuration resembling the circular panoramic model which results in a particular non-central projection allowing the stitching of a non-central panorama. From a single panorama we obtain well-conditioned 3D reconstruction of lines, which are specially interesting in texture-less scenarios. No previous information about the direction or arrangement of the lines in the scene is assumed. The proposed method is evaluated on both synthetic and real images.

(c) 2017 Elsevier Ltd. All rights reserved.

The final publication is available at http://dx.doi.org/10.1016/j.patrec.2017.05.006.

\section{Introduction}

In central imaging systems any projection ray intersects a single point usually known as optical center. In the last decade, the need of a wider field of view increased the development of new imaging systems known as omnidirectional systems allowing complete panoramic images. For a better understanding of the images most of these systems were sought to be central (e.g. the paracatadioptric system, the hypercatadioptric system Baker and Nayar (2001), multicamera systems with common optical center Kim et al. (2008)). The advantage of central systems is that they also allow using previous standard algorithms. However, some of the proposed omnidirectional systems were non-central by construction (e.g. conical catadioptric systems, spherical catadioptric systems). In these systems, rays do not intersect in a single optical center, hence they can not be defined by a direction vector and require richer descriptions like the generalized camera model Grossberg and Nayar (2001) based on Plücker lines Pless (2003); Miraldo et al. (2011) and Lee et al. (2015). Although the higher complexity is a disadvantage, the properties of line projections in non-central systems can turn this disadvantage into an advantage.

\footnotetext{
${ }^{* *}$ Corresponding author: Tel.: +34 87655 5075;

e-mail: bermudez@unizar.es (Jesus Bermudez-Cameo)
}

The projection surface of a 3D line in central systems is a plane. All lines lying in this plane share the same line-image because only two of the four degrees of freedom (DOF) of the 3D line are preserved in the projection. Hence, the only way to recover the direction of the lines and depth up to scale from a single view is imposing additional constraints like dominant directions or perpendicularity Ramalingam and Brand (2013). By contrast, in non-central cameras the projection surface of a line encapsulates the four degrees of freedom of the 3D line. Hence, the geometry of a 3D line can be recovered from a single projection (see Fig. 11. In particular, four points on a line-projection (except some degenerate cases) define four projecting rays providing four independent constraints for computing the complete geometry of the line Teller and Hohmeyer (1999). Unfortunately, this approach is difficult to implement in practice. The quality of the extracted 3D line depends on a magnitude we call the effective baseline of a set of rays which is related with the distances between the four defining skew rays. This effective baseline is too small when using non-central catadioptric systems, so for exploiting this property in practice the system has to be large, Lee et al. (2015).

The motivations of using line-features in non-central systems are numerous. As in central systems, line-features are useful for reconstructing texture-less scenarios. Besides, in noncentral systems metric reconstruction of 3D lines can be recovered from a single panoramic view and no assumption about 

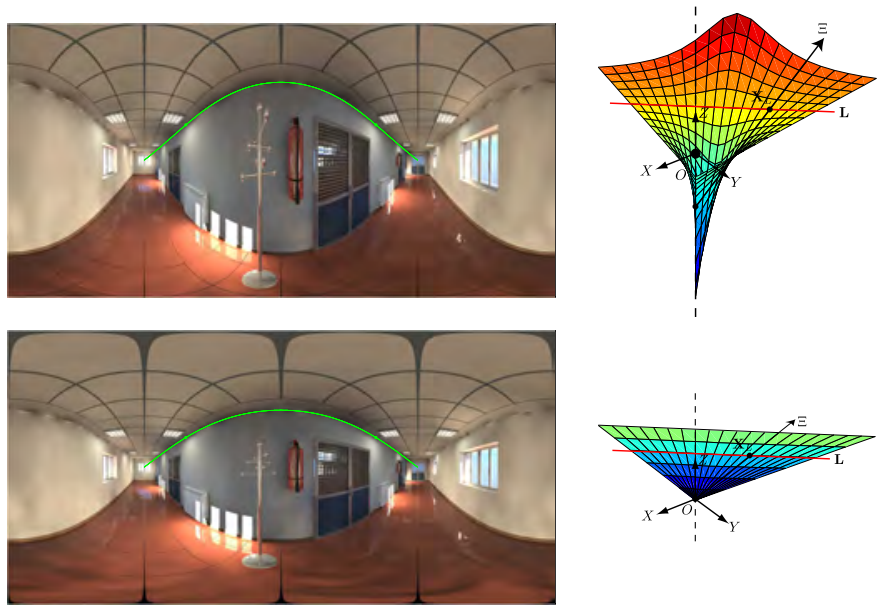

Fig. 1. Top: Line projection in a circular panoramic image with $R_{c}=0.5 \mathrm{~m}$ (Non-central). The line-projection (in green in Top-left) corresponds to the ruled projection surface (Top-right) intersecting only two lines not being projecting rays: the red line and the revolution axis of the system. Bottom: Line projection in a circular panoramic image with $R_{c}=0 \mathrm{~m}$ (A central spherical panoramic image). The line-projection (in green in Bottom-left) corresponds to a planar projection surface (Bottom-right) which is going to be the same projection surface for any $3 \mathrm{D}$ line contained in the plane.

the direction or the arrange of the 3D lines is needed. In addition, considering sequences of images, long lines can be seen along large fragments of the sequence reducing the drift in pose estimation.

\subsection{Previous work}

The geometry of non-central systems has previously been studied for computer vision applications. The back projection of different non-central catadioptric systems is introduced in Swaminathan et al. (2006). Contrary to the back projection case, the forward projection of non-central systems does not necessarily have a closed form solution. Polynomial solutions for the forward projection models in axial non-central catadioptric systems based on quadric mirrors are compiled in Agrawal et al. (2011). In López-Nicolás and Sagüés (2014) the epipolar geometry of conical catadioptric systems and its corresponding calibration are presented.

Another way to obtain non-central images are images generated from moving cameras without common viewpoint. The epipolar geometry is studied for the case of linear pushbroom camera in Gupta and Hartley (1997) and for the case of noncentral circular panorama in Menem and Pajdla (2004). A multi-camera system can be also tackled as particular case of a non-central system. Lee et al. (2015) propose a new method to solve the pose estimation in a multi-camera system represented by a set of ray bundles in a non-central description using Plücker coordinates.

As previously said, in a non-central image the geometry of 3D lines can be recovered from a single line projection. The fundamentals of this approach are exposed in Teller and Hohmeyer (1999) where it is shown that two lines are the intersection of four generic lines. In Gasparini and Caglioti (2011) this approach is exploited to compute 3D lines from 4 rays in non-central catadioptric systems. Different computation methods are considered and the degeneracies and singular configurations are studied. In Lanman et al. (2006) the approach is used with spherical catadioptric mirrors, and in addition two noncentral systems are used for reconstruction. Work in Swaminathan et al. (2008) extends the approach to planar curves. In Bermudez-Cameo et al. (2014b) the approach is derived to the case of conical catadioptric systems obtaining both the 3D line and the mirror geometry. To improve the accuracy in reconstruction using catadioptric systems some approaches have been proposed: considering only horizontal lines Pinciroli et al. (2005); Chen et al. (2011), exploit cross-ratio properties by Perdigoto and Araujo (2012), imposing constraints like parallelism or perpendicularity by Bermudez-Cameo et al. (2014a) or imposing prior information about the gravity direction by Bermudez-Cameo et al. (2016). Using off-axis systems Caglioti et al. (2007) allows avoiding the degeneracies caused by the revolution symmetry. As application, the pose of non-central catadioptric systems is estimated in an image sequence Miraldo et al. (2015) using known 3D lines.

\subsection{Open issues}

The underlying difficulties of line-image fitting and the low accuracy with current non-central systems have left a considerable list of open issues involving non-central 3D line reconstruction. That includes automatic line-image extraction, lineimage tracking, visual odometry and calibration.

\subsection{Contributions}

In this paper, we rediscover the circular panoramic imaging system as a non-central system, Menem and Pajdla (2004), and we propose it through a multi-camera configuration as a way to have enough effective baseline for a practical application of 3D line reconstruction from a single non-central line projection Gasparini and Caglioti (2011). We present a new method for automatic line extraction valid for the proposed non-central system. The result is a metric reconstruction of lines-based scenarios from a single panorama without making assumptions about the direction or the arrangement of the lines. The noncentral circular panoramic system is compared with other catadioptric non-central systems to evaluate the proposed system advantages. We also propose a multi-camera architecture for obtaining an approximation of a circular panorama without the disadvantages of a moving camera. The extraction method is then particularized to the multi-camera case. The methods are tested on realistic simulated scenarios and on real images.

\section{Non-central circular panorama model}

In this section, we describe the projection model of noncentral circular panoramas. A circular imaging panoramic projection, Menem and Pajdla (2004); Gasparini and Caglioti (2011), is a projection model with symmetry of revolution in which any projecting ray intersects both an axis of revolution and a circle of a given radius $R_{c}$ (see Fig. 2). A camera configuration fulfilling this constraint is a linear sensor turning around an axis of revolution. The resulting image of this system is a 


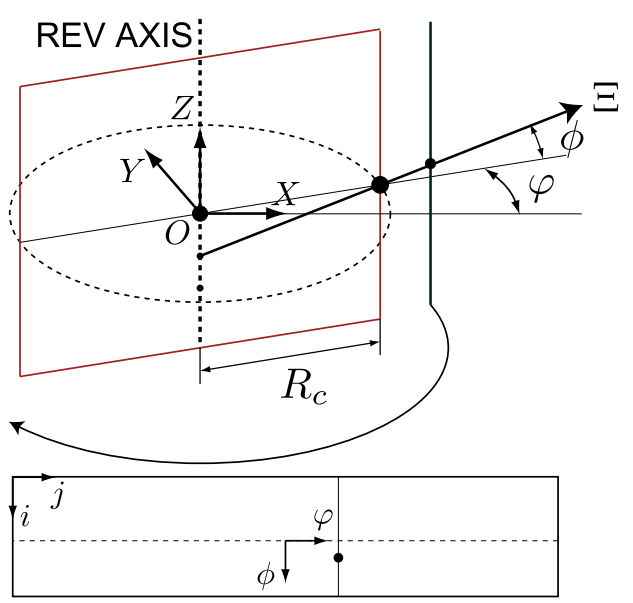

Fig. 2. Non-central circular imaging projection system. Projecting rays intersect a circle of radius $R_{c}$ and the vertical axis. The image coordinates of a pixel $(j, i)$ are linearly related with the azimuth angle $\varphi$ and the elevation angle $\phi$ of the corresponding ray $\Xi$.

panoramic image in which the axis of coordinates are a scaled representation of two angles $\phi, \varphi$ which are the spherical coordinates of the direction vector of each projecting ray. The forward and back projection models of a circular panoramic system have analytical solution and its computation is not time consuming.

\subsection{Back projection model}

Consider the angles $\phi$ and $\varphi$, which are related with the image coordinates $(j, i)$ as follows (see Fig. 2p,

$$
\varphi=j \frac{\varphi_{\text {end }}-\varphi_{\text {ini }}}{n_{\text {columns }}}+\varphi_{\text {ini }}, \quad \phi=i \frac{\phi_{\text {end }}-\phi_{\text {ini }}}{m_{\text {rows }}}+\phi_{\text {ini }}
$$

where $\left(m_{\text {rows }}, n_{\text {columns }}\right)$ are the dimensions of the panorama and $\left(\varphi_{\text {ini }}, \varphi_{\text {end }}\right)$ and $\left(\phi_{\text {ini }}, \phi_{\text {end }}\right)$ are the limits of the field of view of the imaging system. The back projection model can be described by the definition of each projecting ray with Plücker coordinates $\boldsymbol{\Xi}=\left(\boldsymbol{\xi}^{\top}, \overline{\boldsymbol{\xi}}^{\top}\right)^{\top}$ where

$$
\xi=\left(\begin{array}{c}
\cos \phi \cos \varphi \\
\cos \phi \sin \varphi \\
\sin \phi
\end{array}\right), \quad \bar{\xi}=\left(\begin{array}{c}
R_{c} \sin \phi \sin \varphi \\
-R_{c} \sin \phi \cos \varphi \\
0
\end{array}\right)
$$

\subsection{Forward projection model}

Given a 3D point in homogeneous coordinates $\mathbf{X}=$ $\left(x_{1}, x_{2}, x_{3}, x_{0}\right)^{\top}$ the forward projection is computed as follows

$$
\begin{gathered}
\varphi=\operatorname{atan} 2\left(x_{2}, x_{1}\right), \quad \phi=\arctan \frac{x_{3}}{\sqrt{x_{1}^{2}+x_{2}^{2}}-x_{0} R_{c}} \\
j=n_{\text {columns }} \frac{\varphi-\varphi_{\text {ini }}}{\varphi_{\text {end }}-\varphi_{\text {ini }}}, \quad i=m_{\text {rows }} \frac{\phi-\phi_{\text {ini }}}{\phi_{\text {end }}-\phi_{\text {ini }}}
\end{gathered}
$$

\section{Line estimation in non-central systems}

First we present the procedure for 3D line estimation in noncentral systems. Secondly we present the expression for the line-image in non-central circular panoramas. Using Plücker coordinates, the intersection between lines is described by the side operator (we use the same notation as in Pottmann and Wallner (2001)). A given line $\mathbf{L}=\left(\mathbf{I}^{\top}, \mathbf{I}^{\top}\right)^{\top} \in \mathbb{P}^{5}$ (where $\mathbf{l} \in \mathbb{R}^{3}$ and $\mathbf{I} \in \mathbb{R}^{3}$ ) intersects a ray $\boldsymbol{\Xi} \in \mathbb{P}^{5}$ if

$$
\operatorname{side}(\mathbf{L}, \boldsymbol{\Xi})=\mathbf{L}^{\top} \mathbf{W} \boldsymbol{\Xi}=\mathbf{l}^{\top} \overline{\boldsymbol{\xi}}+\overline{\mathbf{I}}^{\top} \boldsymbol{\xi}=0
$$

where $\mathrm{W}=\left(\begin{array}{cc}0_{3 \times 3} & I_{3 \times 3} \\ I_{3 \times 3} & 0_{3 \times 3}\end{array}\right)$.

Since a 3D line has four degrees of freedom we need at least 4 equations to solve for $\mathbf{L}$. If four projection rays from a 3D line provide four independent constraints we can compute the 3D line from the system of equations Teller and Hohmeyer (1999)

$$
A \mathbf{L}=\mathbf{0},
$$

where $\mathrm{A}_{i}=\left(\begin{array}{cc}\bar{\xi}_{i}^{\top} & \xi_{i}^{\top}\end{array}\right)$ is the $\mathrm{A}_{i}$ row of the matrix A.

Plücker coordinates is an over-parametrized representation of a line in $\mathbb{P}^{5}$ and the solution of $(6)$ is a one-dimensional subspace of $\mathbb{P}^{5}: \mathbf{L}=\mathbf{L}_{0}+\lambda \mathbf{L}_{1}$ described by two elements of $\mathbb{P}^{5}, \mathbf{L}_{0}$ and $\mathbf{L}_{1}$ which can be computed using Singular Value Decomposition. Imposing the Plücker identity $\left(\mathbf{l}^{\top} \overline{\mathbf{I}}=0\right)$ we obtain the intersection with the Klein Quadric Pottmann and Wallner(2001) which results in two solutions. One of them is the sought 3D line. If the system is axial the other solution is the axis of revolution. In some degenerate cases four rays do not provide four independent equations Gasparini and Caglioti (2011): when the projection surface is a plane (the line is coplanar with the axis of revolution or coplanar with the plane containing the circle) or when the projection surface is a regulus.

\subsection{Line-image in non-central circular panorama}

Deriving (5) for circular panoramic systems the homogeneous expression for the line-image on the panoramic image becomes

$$
\begin{array}{r}
\cos \phi\left(\bar{l}_{1} \cos \varphi+\bar{l}_{2} \sin \varphi\right)+\ldots \\
\sin \phi\left(\bar{l}_{3}+R_{c}\left(l_{1} \sin \varphi-l_{2} \cos \varphi\right)\right)=0
\end{array}
$$

which allows estimating the line-image from four image points $\left(\varphi_{i}, \phi_{i}\right), i=1 . .4$. Due to the symmetry of revolution of the system $\left(\bar{\xi}_{3}=0\right), l_{3}$ is not present in (7) and the solution is an element of $\mathbb{P}^{4}$. In this case the null space gives one solution and Plücker identity $\left(\mathbf{I}^{\top} \mathbf{I}=0\right)$ gives $l_{3}=-\frac{l_{1} \bar{l}_{1}+l_{2} \bar{l}_{2}}{\bar{l}_{3}}$.

Equation (7) also allows a parametric representation expressing $\phi$ in terms of $\varphi$ (see Fig. 11):

$$
\tan \phi=\frac{-\left(\bar{l}_{1} \cos \varphi+\bar{l}_{2} \sin \varphi\right)}{\bar{l}_{3}+R_{c}\left(l_{1} \sin \varphi-l_{2} \cos \varphi\right)}
$$

Since we know the location of the 3D line we can also compute its vanishing points by projecting the direction onto the image. 


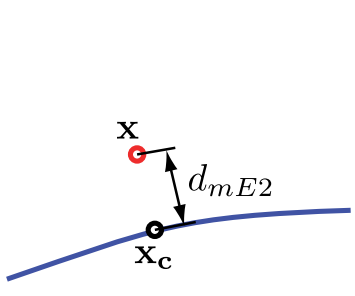

(a)

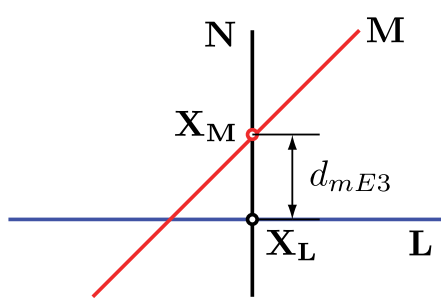

(b)
Fig. 3. Distance to a line-image. (a) Distance to a line projection on the image. (b) Distance between a ray $M$ and a 3D line $L$.

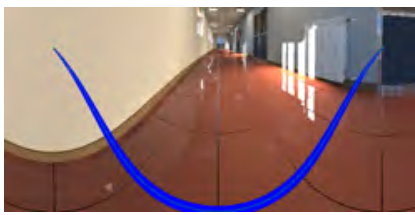

(a)

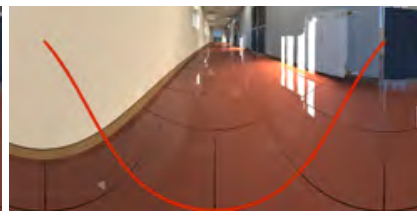

(b)
Fig. 4. Region around a line projection defined by a given distance. (a) Metric distance $\mathbb{E}^{3}$ from ray to line. (b) Proposed metric distance on the image.

\section{Distance from points to line-images}

In this section we propose a distance measurement to evaluate when an image point belongs to a line-image. It also can be used to optimize the line estimation in an optimization scheme. Depending on the used distance we will reach different results.

The Euclidean metric distance in $\mathbb{E}^{3}$ is the minimum $\mathrm{Eu}$ clidean distance between two 3D lines (the 3D line and the projecting ray generated from an image point). This distance is defined as in Pottmann and Wallner (2001):

$$
d_{m E 3}=\frac{\left|\mathbf{l}_{i}^{\top} \overline{\mathbf{l}}_{j}+\mathbf{l}_{j}^{\top} \overline{\mathbf{l}}_{i}\right|}{\left\|\mathbf{l}_{i} \times \mathbf{l}_{j}\right\|} .
$$

When determining if a point lies on a line-image in a RANSAC or in a Hough transform scheme this distance tends to give higher reward to lines which are close to the origin. This effect induces errors in the extraction process.

To avoid this effect the metric distance $\mathbb{E}^{2}$ from point to lineimage can be used. This is performed by using the method of Lagrange multipliers to obtain the point of the line-image $\mathbf{x}_{c}$ which is closer to the given point $\mathbf{x}$ and computing the Euclidean distance between them (see Fig. 3 (a)). However, except for some particular cases Bermudez-Cameo et al. (2014b), this approach does not have a closed form solution and needs to be solved iteratively. We propose in this paper to compute the points on the $3 \mathrm{D}$ lines $\left(\mathbf{X}_{L}\right.$ and $\left.\mathbf{X}_{M}\right)$ which have the minimum metric distance $d_{m E 3}$ in $\mathbb{E}^{3}$ (see Fig. $3 \mathrm{~b}$ ). Then, instead of using distance in $\mathbb{E}^{3}$ we propose to project both points and compute the metric $\mathbb{E}^{2}$ distance on the image. The advantage is that this computation has a closed form and can be used in any non-central system in which the forward projection has a closed solution. Notice that the projection of the closest point in the 3D space $\mathbf{X}_{M}$ is not exactly the same as the closest point $\mathbf{x}_{c}$ on the image.

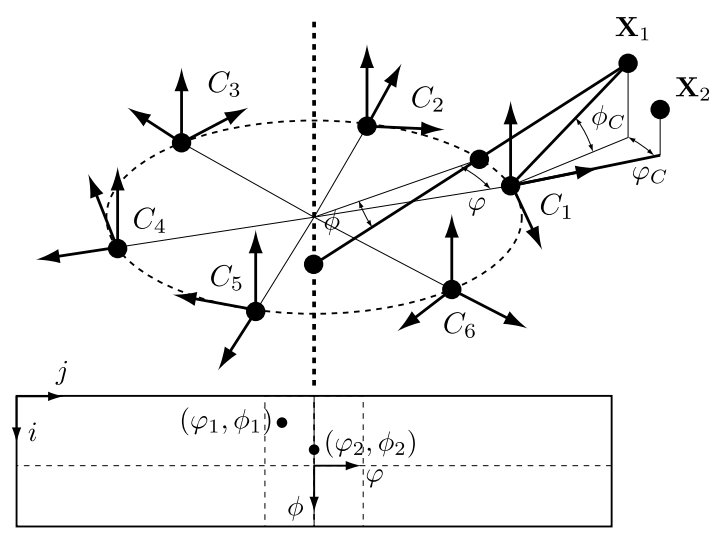

Fig. 5. Multi-camera system with 6 cameras arranged in a circle.

To compute these closest points we have to compute a third line passing through both lines and with direction orthogonal to the directions of both 3D lines (see Fig. 3 b). Given a line $\mathbf{L}=\left(\mathbf{I}^{\top}, \overline{\mathbf{I}}^{\top}\right)^{\top}$ and other line $\mathbf{M}=\left(\mathbf{m}^{\top}, \overline{\mathbf{m}}^{\top}\right)^{\top}$ representing the projection ray of a given point on the image we want to compute a third line $\mathbf{N}=\left(\mathbf{n}^{\top}, \overline{\mathbf{n}}^{\top}\right)^{\top}$ which intersects both lines and is orthogonal to $\mathbf{L}$ and $\mathbf{M}$. To enforce the orthogonal constraint we define $\mathbf{n}=\mathbf{l} \times \mathbf{m}$. The intersections are imposed using the side operator (5). Finally, we obtain a third equation by imposing $\mathbf{N}$ to be a line with the Plücker constraint. The result is a system of three linear equations

$\overline{\mathbf{l}}^{\top}(\mathbf{l} \times \mathbf{m})+\mathbf{l}^{\top} \overline{\mathbf{n}}=0, \quad \overline{\mathbf{m}}^{\top}(\mathbf{l} \times \mathbf{m})+\mathbf{m}^{\top} \overline{\mathbf{n}}=0, \quad \overline{\mathbf{n}}^{\top}(\mathbf{l} \times \mathbf{m})=0$

which is solved for $\overline{\mathbf{n}}$ obtaining

$$
\overline{\mathbf{n}}=\left(\begin{array}{c}
\mathbf{l}^{\top} \\
\mathbf{m}^{\top} \\
(\mathbf{l} \times \mathbf{m})^{\top}
\end{array}\right)^{-1}\left(\begin{array}{c}
-\overline{\mathbf{l}}^{\top}(\mathbf{l} \times \mathbf{m}) \\
-\overline{\mathbf{m}}^{\top}(\mathbf{l} \times \mathbf{m}) \\
0
\end{array}\right)
$$

Once we have computed the line $\mathbf{N}$, we can compute the intersection between $\mathbf{N}$ and $\mathbf{L}$ by using the meet operator obtaining the points

$$
\mathbf{X}_{L}=\left(\begin{array}{c}
-\overline{\mathbf{n}} \times \overline{\mathbf{l}} \\
\mathbf{n}^{\top} \overline{\mathbf{l}}
\end{array}\right), \quad \mathbf{X}_{M}=\left(\begin{array}{c}
-\overline{\mathbf{n}} \times \overline{\mathbf{m}} \\
\mathbf{n}^{\top} \overline{\mathbf{m}}
\end{array}\right)
$$

that are projected on the image using the forward projection model. Then, the metric distance $\mathbb{E}^{2}$ between the projections is computed. In Fig. 4 we compare the region defined by a given distance using the metric distance $\mathbb{E}^{3}$ from ray to line and the proposed metric distance $\mathbb{E}^{2}$ on the image.

Regarding the linear system (6) the intuitive expected behaviour is that using an optimization procedure with (9) or the proposed metric distance $\mathbb{E}^{2}$ on the image can improve the results, but at the end the effective baseline of the rays is determining in the quality of the results. In particular, the further the line the lesser the effective baseline, and in this case the optimization not necessarily improves the result. 

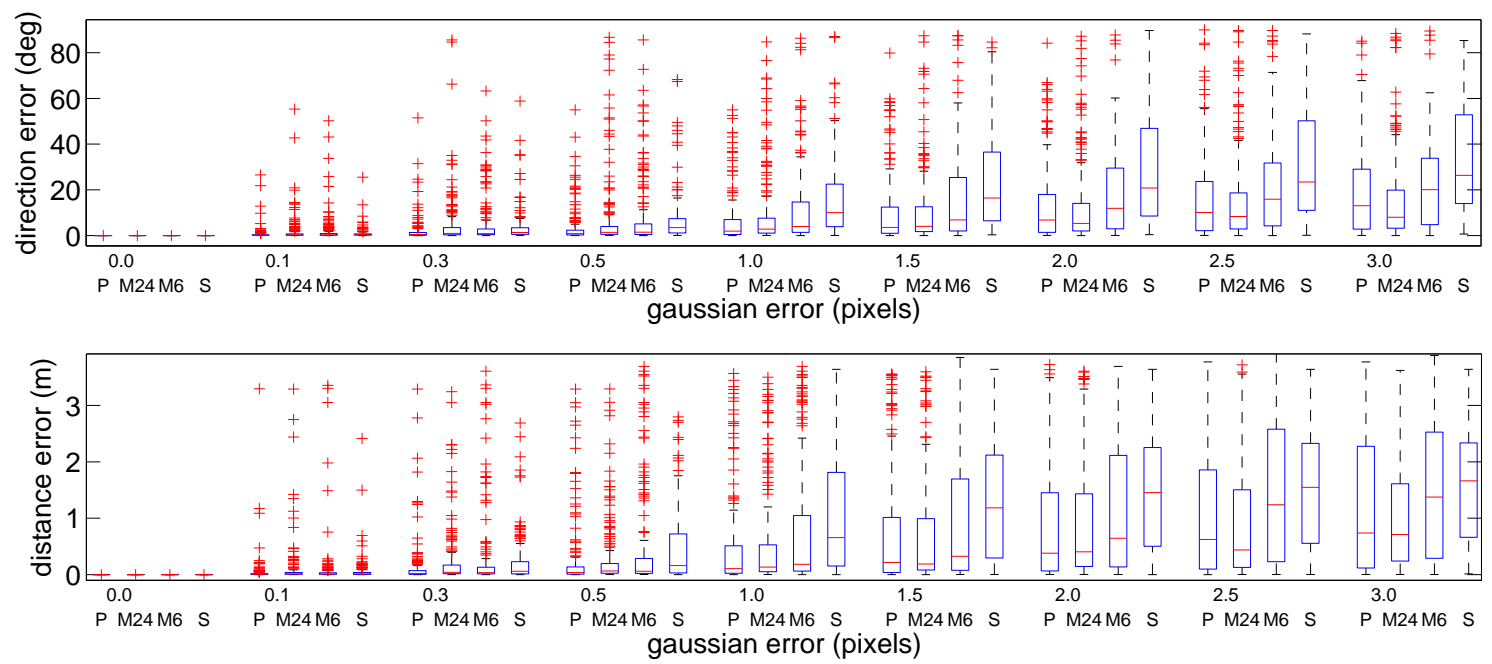

Fig. 6. Comparison in accuracy reconstructing lines using different non-central systems. Top: Direction error in degrees. Bottom: Distance error in meters. $P$ for circular panoramic system. M6 and M24 for a multi-camera having 6 and 24 single fisheye cameras, respectively. S for spherical catadioptric system.

\section{Non-central circular panoramas from Multi-Camera system}

In this section, we propose to model a multi-camera system as a non-central circular panoramic imaging system and we discuss their similarities and differences. We also compare and evaluate the accuracy of $3 \mathrm{D}$ line reconstruction on different non-central camera systems.

A non-central circular panorama can be obtained using an off-centred rotating camera where only the central column of the image is used to stitch the panoramic image. However, this set-up has the synchronization difficulties of a moving camera especially if the whole system is in motion. Instead, it is possible to approximate this configuration with an array of cameras arranged in a circle. With this configuration the coordinates of the projecting ray $\Xi=\left(\boldsymbol{\xi}^{\top}, \overline{\boldsymbol{\xi}}^{\top}\right)^{\top}$ of a point $\mathbf{X}=\left(\mathbf{x}^{\top}, x_{0}\right)^{\top}$ through a camera $\mathrm{i}$ with optical center $\mathbf{C}_{i}=\left(\mathbf{c}_{i}{ }^{\top}, 1\right)^{\top}$ is

$$
\boldsymbol{\xi}=\left(\mathbf{x}-x_{0} \mathbf{c}_{i}\right), \quad \overline{\boldsymbol{\xi}}=\left(\mathbf{c}_{i} \times \mathbf{x}\right)
$$

Consider the projection of point $\mathbf{X}_{1}$ in Fig. 5. The angular coordinates of point $\mathbf{X}_{1}$ in a non-central panorama $(\varphi, \phi)$ can be approximated by the azimuth and elevation angle of the corresponding projecting ray in a conventional camera $\left(\varphi_{C}, \phi_{C}\right)$. This approximation is done in non-overlapped columns covered by the set of cameras. Then, the non-central circular panorama and the proposed multi-camera panorama are equivalent in the columns corresponding with the azimuth of the cameras focal axis (e.g. point $\mathbf{X}_{2}$ in Fig. 5). The rest of the panorama is only perfectly equivalent if the scene is at infinity (e.g. point $\mathbf{X}_{1}$ in Fig. 5). From a practical point of view, only a small part of the center columns of the image is extracted for composing the panorama so the needed bandwidth to acquire the whole set of images reduces considerably. The multi-camera panorama is finally stitched using alpha blending.

\subsection{Multi-camera vs. circular panorama}

The whole system is non-central because the projecting rays do not intersect in a single view-point. However, the proposed multi-camera system is locally central and therefore rays from a single camera can only provide two independent equations to fit a line. This local centrality must be taken into account when getting random points lying on a line projection on the panoramic image.

Consider a random picking of four rays from the panoramic image. Three cases correspond to valid configurations: each ray is picked from a different camera $([1+1+1+1])$, two rays are from the same camera and the two other rays are from two different cameras $([2+1+1])$, lastly two rays are selected from two different cameras $([2+2])$. The other two cases correspond to non-valid configurations: for rays from a single camera ([4]) and three rays from one camera and one ray from another camera $([3+1])$.

There is another difference between the multi-camera system and the circular panoramic imaging system. The circular panoramic system is axial meaning that all the projecting rays intersect the same rotation axis. When computing a 3D line from 4 rays we obtain two solutions, one of them is the sought line and the other is the axis of revolution. However, a multicamera system is not axial and therefore there is not a single line intersecting the projecting rays which would help to discriminate the correct solution. For the case in which we take 2 rays from two cameras one of the solutions must intersect the line defined by the two optical centres, therefore it must lie in the horizontal plane passing through all the optical centres. In other case, we have to use an additional criterion to distinguish between both solutions. In particular, we include both solutions as hypothesis in the RANSAC scheme. Only the correct solution has supporting points on the image, whereas the other solutions are directly rejected in the extraction process.

\subsection{Accuracy of $3 D$ lines in non-central systems}

In this section we evaluate the accuracy of the proposed system for single-view 3D line extraction in comparison to non- 


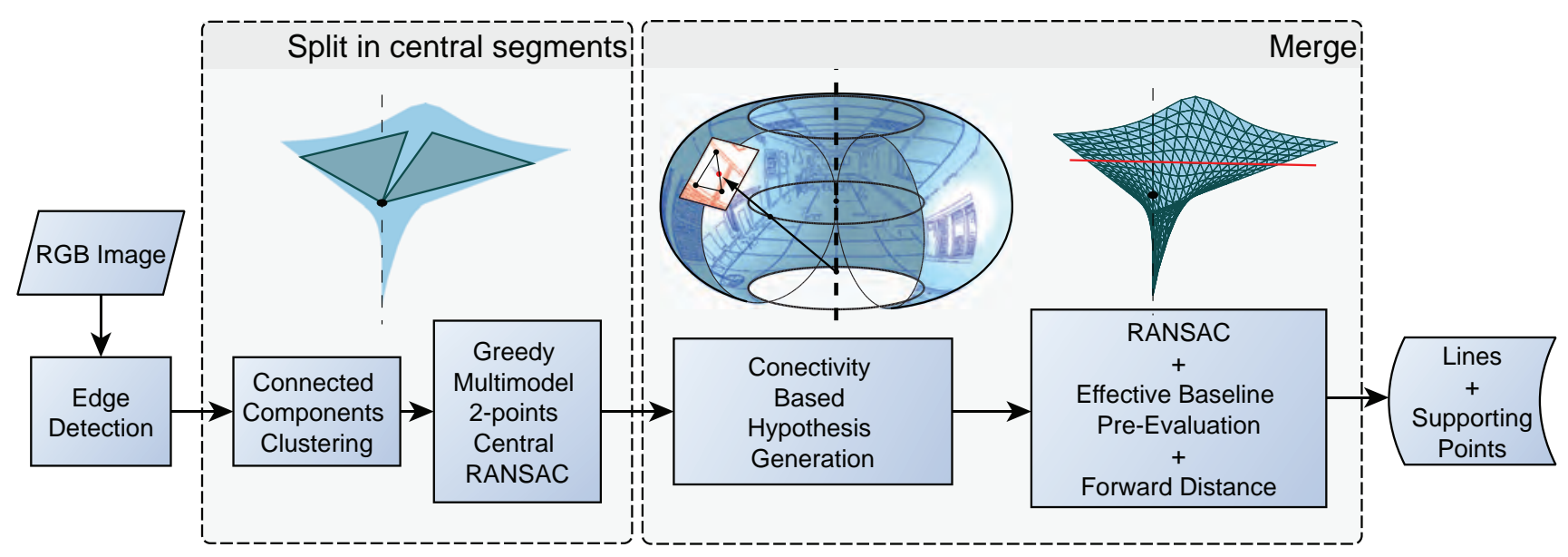

(a)

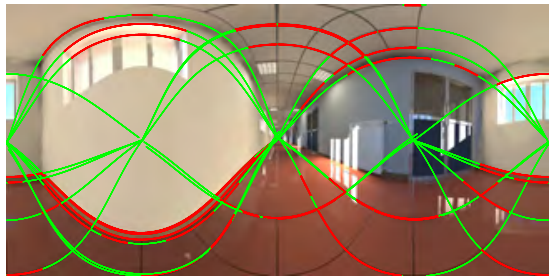

(b) Horizontal

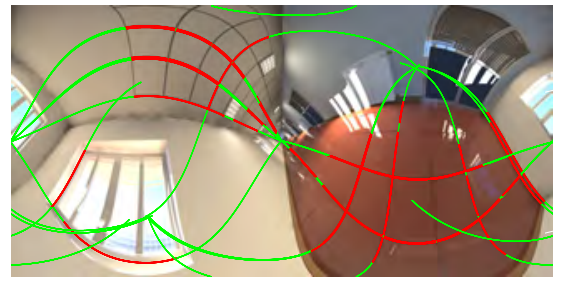

(c) Slanted

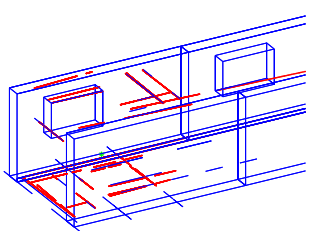

(d) Horizontal

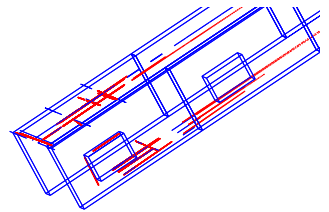

(e) Slanted

Fig. 7. Line extraction in non-central circular panorama. (a) Procedure flowchart. (b,c) Examples of extracted line-images in green and supporting points in red. (d,e) 3D reconstruction (in red) obtained from the back projection of the supporting points of the line-images to the extracted 3D lines.

central catadioptric systems. We assume the points of the image belong to a given line projection. The geometric limitations of reconstructing 3D lines from a single line projection in noncentral systems are given by the relation between the size of the system and the depth of the line (assuming the line is long enough). That means that in most known non-central systems this limitation prevents the practical usage of this method, because the estimated 3D lines are too noisy. However, it also depends on the system type. In this analysis we are considering random lines with a depth up to 3 meters. The length of the lines is $20 \mathrm{~m}$. Four different systems are compared:

- A spherical catadioptric system composed of a spherical mirror with a radius of $1.2 \mathrm{~m}$ and a perspective camera located at $1.8 \mathrm{~m}$ from the center of the sphere. The resolution of the camera is $4096 \times 4096$ pixels.

- A circular panoramic imaging system defined by a circle of radius of $0.3 \mathrm{~m}$. The image has a resolution of $4000 \times 8000$ pixels.

- Two cases of the proposed multicamera system. The first one is composed of 6 fisheye cameras located in a circle of radius $0.3 \mathrm{~m}$. The second one is composed of 24 fisheye cameras in a circle of radius $0.3 \mathrm{~m}$. In both cases the cameras have a resolution of $768 \times 1024$ pixels.

Once the points of the random lines are projected on the corresponding images, we add Gaussian noise of a given $\sigma$. We variate the value of $\sigma$ from 0 to 1 pixel. In Fig. 6 we compare the results. Fig. 6 Top depicts the distribution of the direction error (degrees) of the estimated 3D lines with respect to the ground truth. Fig. 6 Bottom shows the distribution of the distance error. Both the circular panoramic system and multicameras clearly outperforms in terms of accuracy the classical catadioptric non-central systems despite having smaller sizes (0.3 m vs $1.2 \mathrm{~m})$.

\section{Line-extraction in non-central panoramas}

The extraction of line projections in non-central systems is still an open topic. In this section we present our proposal for extracting lines in non-central circular panoramas also used in the multi-camera panoramas obtained from real images.

First the panoramic image is preprocessed using a Rolling Guidance Filter proposed by Zhang et al. (2014) to reduce textured patterns but conserving the edges. Then, the edges in the image are extracted using Canny detector. After that, we propose a split and merge approach. First, edges are stored in connected components. To reduce the complexity of the line extraction we first estimate locally central approximations of the line projection. The goal is obtaining consistent segments of line-image fitting planes that locally describe the projection surface (see Fig. 7(a)). A greedy multi-model RANSAC is executed on each connected component obtaining a set of central segments. As input for this central RANSAC we use central approximations obtained from the direction of the real projection rays (defined by (2) in the case of the non-central panorama 


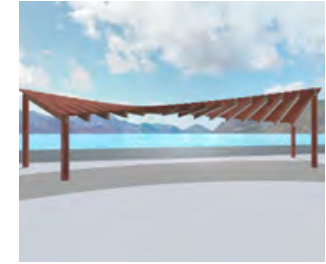

(a)

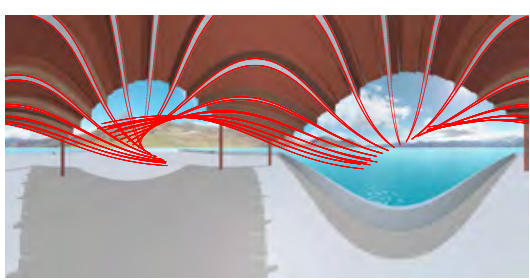

(b)

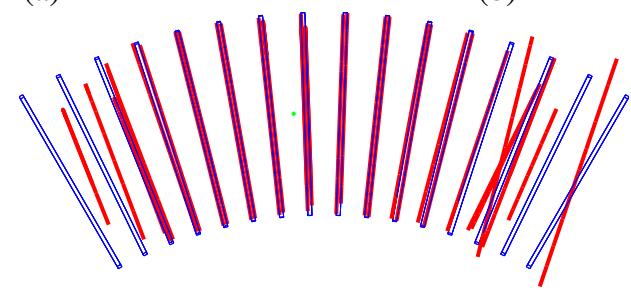

(c)

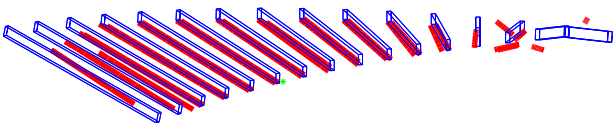

(d)

Fig. 8. Reconstruction of a non-Manhattan scene from a non-central circular panorama. (a) Perspective view of the scenario and (b) non-central panorama. 3D reconstruction: (c) Top view. (d) Orthografic view.

and by (13) when using a multi-camera system). For merging the central segments we are interested on the connectivity among them. In the non-central panorama the locus of the optical center is a circle. The orthogonal space to the collection of projecting rays is then a family of toroidal surfaces. The connectivity map is obtained by discretizing the torus in a set of tangent planar patches where neighbours are projected using orthographic mapping (see Fig. 7)(a)). Then the local connectivity of the segments is estimated with a Delaunay triangulation. The resulting sparse connectivity matrix is a composition of these local connections. Then, the connectivity of the central segments is exploited to generate a collection of hypotheses in a USAC framework, by Raguram et al. (2013). A set of different combinations of three segments is randomly generated. All the combinations not satisfying the connectivity are removed. Then, the remaining hypotheses are evaluated. Each hypothesis is tested using the distance presented in Section 4 and taking into account the effective baseline of each set of rays. To measure the effective baseline of a set of $n$ rays we use a function $z_{b s}$ depending on the metric distances $d_{i j} \in \mathbb{E}^{3}$ between the defining rays (unless degenerate cases projecting rays are skew in circular panoramic systems)

$$
z_{b s}=\frac{n !}{2(n-2) !}\left(\sum_{i=1}^{n-1} \sum_{j=i+1}^{n} \frac{1}{d_{i j}}\right)^{-1} .
$$

This function increases when the distances between rays increase but also avoids that any individual distance $d_{i j}$ turns to zero. The results of the extraction method are the 3D lines and the corresponding edges supporting the line-images that are directly related with the $3 \mathrm{D}$ points on the $3 \mathrm{D}$ line (see Fig. 7 (b-e)).
Table 1. Error distribution in line-image extraction.

\begin{tabular}{|l|c|c|c|c|}
\hline \multirow{2}{*}{ Scenario } & \multicolumn{2}{|c|}{ angular error (degs) } & \multicolumn{2}{c|}{ depth error $(\mathrm{m})$} \\
\cline { 2 - 5 } & median & $\sigma$ & median & $\sigma$ \\
\hline Horizontal & 0.948 & 1.180 & 0.028 & 0.036 \\
Slanted & 0.954 & 1.105 & 0.091 & 0.126 \\
\hline
\end{tabular}

\section{Experimental evaluation}

To evaluate the proposed extraction method we have performed experiments with synthetic and real images. Synthetic images have been generated using a spin-off version of Pov-Ray called Mega-Pov which allows to define the non-central circular panoramic camera as a parametric camera. The scenario is a modification of a publicly available synthetic scenario 1 The synthetic panoramas have resolutions of $4096 \times 2048$ pixels and the radius of the generation circle is $R_{c}=0.5 \mathrm{~m}$. In Fig. 7 (b,c) we show some examples of extracted lines from synthetic panoramas. The extracted line-images are depicted in green and the edge points supporting the line-image in red. The corresponding 3D reconstruction is shown in Fig. 7(d) where the ground truth is coloured in blue and the reconstructed segments are depicted in red. One degenerate case is when the line lies in a plane passing through the axis of revolution of the system (the projected line-image is a straight $2 \mathrm{D}$ line). The further a line is from this degeneration the better conditioned it is. Then, horizontal lines are better conditioned than slanted lines. In Fig. 7)(c,e) we show a similar example having slanted lines after rotating the camera 50 degrees. In Table. 1 we show a comparison between the extraction error in both scenarios (horizontal and slanted). Given the error array $e_{i}$ the standard deviation $\sigma$ is robustly estimated using the median absolute deviation $\left(\sigma=1.4286\right.$ median $\mid e_{i}-$ median $\left.\left(e_{i}\right) \mid\right)$.

Just to illustrate that we are not imposing or using Manhattan conditions or dominant directions in Fig. 8 we present an example of reconstruction of a non-Manhattan scenario of a parking with a singular roof. In this particular example the defining points of the line-images have been manually selected showing that there is a limit on the accuracy of the reconstruction which is directly related with the effective baseline of the system and not related with the extraction procedure. In Fig. $8(\mathrm{c}, \mathrm{d})$ we show the fitting and reconstruction of 3D lines of the roof. We can see accuracy in 3D reconstruction decreases in further lines despite being correctly fitted on the image.

\subsection{Experiments with real images}

The real panoramas have been composed using a rotating offaxis fisheye camera (uEye UI-148xSE-C with lens Lensagon CF5M1414) with radius $R_{c}=0.5 \mathrm{~m}$. In Fig 9 we show examples of line-image extraction and $3 \mathrm{D}$ reconstruction from panoramic images of $4096 \times 1570$ pixels. The panorama presented in Fig. 9 (a) has been composed from a stream of 180 images covering each one a step size of 2 degrees and having $40 \times 1920$ pixels.

${ }^{1}$ http://hof.povray.org/office-13.html The Office - J. Vives-Piqueres, 2004 


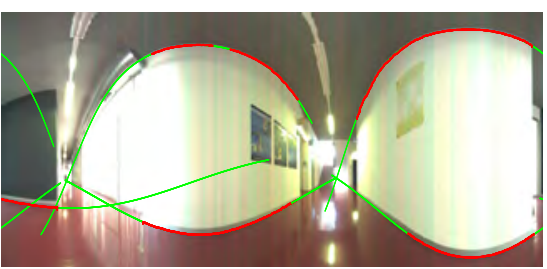

(a) 2 deg of step size

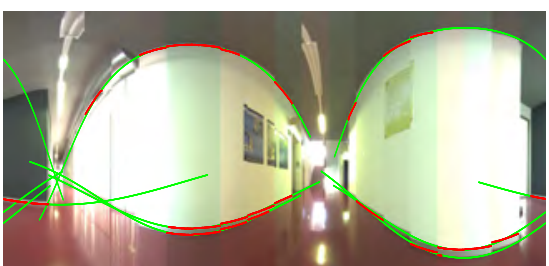

(b) 18 deg of step size

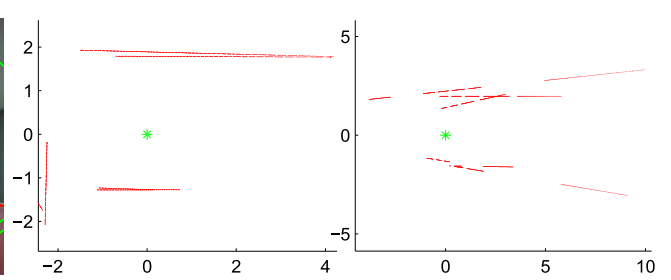

(c) 2 deg of step size (d) 18 deg of step size

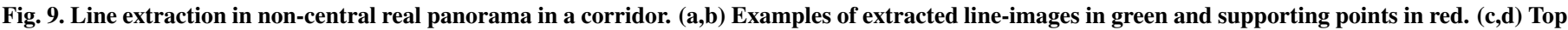
view of the obtained 3D reconstruction (in red). The green cross represents the camera location.

In contrast, the panorama shown in Fig. 9(b) has been stitched from a stream of 20 images covering each one 18 degrees of field of view with $360 \times 1920$ pixels. For evaluating the reconstruction we have measured the environment. The width of the corridor measured with a laser electronic distance meter (EDM) is $3.20 \mathrm{~m}$. From the circular panorama shown in Fig. 9 (a) we obtain a measure of $3.16 \mathrm{~m}$.

\section{Conclusions}

In this paper, we propose a non-central system for 3D reconstruction of lines from single panoramas without assumptions about the direction or arrangement of the lines (such as Manhattan world). We compare our configuration with other known non-central cameras concluding that (in contrast with other non-central systems) our configuration has enough effective baseline to perform 3D reconstruction of a real scenario with typical dimensions. This result opens new possibilities for real applications. We also present a new method for automatic extraction of line-images from non-central circular panoramic images. To the best of our knowledge this is the first algorithm able to obtain an automatic extraction of line-images from noncentral images. The proposal was tested with realistic synthetic images and with real images.

\section{Acknowledgments}

This work was supported by DPI2014-61792-EXP and DPI2015-65962-R (MINECO/FEDER, UE).

\section{References}

Agrawal, A., Taguchi, Y., Ramalingam, S., 2011. Beyond alhazen's problem: Analytical projection model for non-central catadioptric cameras with quadric mirrors, in: Computer Vision and Pattern Recognition, pp. 2993 3000.

Baker, S., Nayar, S.K., 2001. Single viewpoint catadioptric cameras. SpringerVerlag New York, Inc., Secaucus, NJ, USA.

Bermudez-Cameo, J., Barreto, J.P., Lopez-Nicolas, G., Guerrero, J.J., 2014a. Minimal solution for computing pairs of lines in non-central cameras, in: The 12th Asian Conference on Computer Vision.

Bermudez-Cameo, J., Demonceaux, C., Lopez-Nicolas, G., Guerrero, J.J., 2016. Line reconstruction using prior knowledge in single non-central view, in: 27th British Machine Vision Conference (BMVC).

Bermudez-Cameo, J., Lopez-Nicolas, G., Guerrero, J.J., 2014b. Line-images in cone mirror catadioptric systems, in: 22th International Conference on Pattern Recognition.

Caglioti, V., Taddei, P., Boracchi, G., Gasparini, S., Giusti, A., 2007. Singleimage calibration of off-axis catadioptric cameras using lines, in: International Conference on Computer Vision (ICCV), pp. 1-6.
Chen, W., Cheng, I., Xiong, Z., Basu, A., Zhang, M., 2011. A 2-point algorithm for 3D reconstruction of horizontal lines from a single omni-directional image. Pattern Recognition Letters 32, 524-531.

Gasparini, S., Caglioti, V., 2011. Line localization from single catadioptric images. International journal of computer vision 94, 361-374.

Grossberg, M.D., Nayar, S.K., 2001. A general imaging model and a method for finding its parameters, in: International Conference on Computer Vision, pp. 108-115.

Gupta, R., Hartley, R.I., 1997. Linear pushbroom cameras. Pattern Analysis and Machine Intelligence, IEEE Transactions on 19, 963-975.

Kim, J.H., Li, H., Hartley, R., 2008. Motion estimation for multi-camera systems using global optimization, in: IEEE Conference on Computer Vision and Pattern Recognition, pp. 1-8.

Lanman, D., Wachs, M., Taubin, G., Cukierman, F., 2006. Reconstructing a 3D line from a single catadioptric image, in: Third International Symposium on 3D Data Processing, Visualization, and Transmission, pp. 89-96.

Lee, G.H., Li, B., Pollefeys, M., Fraundorfer, F., 2015. Minimal solutions for the multi-camera pose estimation problem. The International Journal of Robotics Research .

López-Nicolás, G., Sagüés, C., 2014. Unitary torus model for conical mirror based catadioptric system. Computer Vision and Image Understanding 126, $67-79$.

Menem, M., Pajdla, T., 2004. Constraints on perspective images and circular panoramas., in: BMVC, pp. 1-10.

Miraldo, P., Araujo, H., Goncalves, N., 2015. Pose estimation for general cameras using lines. IEEE Transactions on Cybernetics , 1-1.

Miraldo, P., Araujo, H., Queiró, J., 2011. Point-based calibration using a parametric representation of the general imaging model, in: International Conference on Computer Vision, pp. 2304-2311.

Perdigoto, L., Araujo, H., 2012. Reconstruction of 3D lines from a single axial catadioptric image using cross-ratio, in: 21th International Conference on Pattern Recognition (ICPR), pp. 857-860.

Pinciroli, C., Bonarini, A., Matteucci, M., 2005. Robust detection of 3D scene horizontal and vertical lines in conical catadioptric sensors, in: Proc. 6th Workshop on Omnidirectional Vision.

Pless, R., 2003. Using many cameras as one, in: Computer Vision and Pattern Recognition, pp. II-587.

Pottmann, H., Wallner, J., 2001. Computational line geometry. Springer.

Raguram, R., Chum, O., Pollefeys, M., Matas, J., Frahm, J., 2013. Usac: a universal framework for random sample consensus. Pattern Analysis and Machine Intelligence, IEEE Transactions on 35, 2022-2038.

Ramalingam, S., Brand, M., 2013. Lifting 3d manhattan lines from a single image, in: International Conference on Computer Vision, pp. 497-504.

Swaminathan, R., Grossberg, M.D., Nayar, S.K., 2006. Non-single viewpoint catadioptric cameras: Geometry and analysis. International Journal of Computer Vision 66, 211-229.

Swaminathan, R., Wu, A., Dong, H., et al., 2008. Depth from distortions, in: The 8th Workshop on Omnidirectional Vision, Camera Networks and Nonclassical Cameras.

Teller, S., Hohmeyer, M., 1999. Determining the lines through four lines. Journal of graphics tools $4,11-22$.

Zhang, Q., Shen, X., Xu, L., Jia, J., 2014. Rolling guidance filter, in: European Conference on Computer Vision, pp. 815-830. 